\title{
Gorter-Mellink pulsed-source problem in cylindrical and spherical geometry*
}

Lawrence Dresner

Oak Ridge National Laboratory

P.O. Box 2009

Oak Ridge, TN 37831-8040
CONF-9108132--1

DE9 017249

\section{Abstract}

An exact solution to the Gorter-Mellink pulsed-source problem is known in plane geometry [Dresner, L. Advances in Cryogenic Engineering 29 (1984) 323]. According to this solution, the central temperature (i.e., the temperature at the source location) falls as $t^{-3 / 2}$, where $t$ is the elapsed time after an instantaneous, plane pulse. No such exact solutions are known to the pulsed-source problem in cylindrical and spherical geometry. But in cylindrical geometry, it can be shown that if the initial condition is an instantaneous temperatue rise $\Delta T$ inside a cylinder of radius $R$, the central temperature is bounded from above by a decreasing exponential function of time. The relaxation time of this exponential is related to $\Delta T$ and $R$. In spherical geometry, it can be shown that if the initial condition is an instantaneous temperature rise $\Delta T$ inside a sphere of radius $R$, the central temperature is bounded from above by a function proportional to $\left(t_{0}-t\right)^{9 / 2}$. The extinction time $t_{0}$ is related to $\Delta T$ and $R$. These predictions have not been tested by experiment. and the author recommends such experiments.

*Research sponsored by the Defense Nuclear Agency, L.S. Department of Defense, under Martin Marietta Energy Systems, Inc., contract DE-AC05-84OR21400 with the U.S. Departmer.t of Energy 


\section{Introduction}

The pulsed-source problem is a problem of heat diffusion in which a plane, line, or point source in an infinite medium is pulsed at $t=0$ and the resulting temperature distribution studied as a function of elapsed time. Depending on which source is used, the pulsed-source problem is said to refer to plane, cylindrical, or spherical geometry or to one, two, or three dimensions, respectively.

If the diffusion is described by the ordinary diffusion equation, $\partial T / \partial t=\nabla^{2} T$, the pulsed-source problem has well-known analytic solutions in which the central temperature falls with elapsed time $t$ as $t^{-n / 2}$, where $n$ is the dimensionality mentioned above.

Heat transport in turbulent He-II (superfluid helium) does not obey the ordinary diffusion equation. The latter equation is based on Fourier's law of conduction, which says that the heat flux is proportional to the gradient of the temperature. Turbulent superfltid helium instead obeys the Gorter-Mellink relation, which says that the heat flux is proportional to the cube root of the gradient of the temperature. For the Gorter-Mellink relation, the diffusion equation takes the form

$$
S \frac{\partial T}{\partial t}=\nabla \cdot\left[K(\nabla T)^{1 / 3}\right]
$$

where $S$ is the heat capacity per unit volume and $K$ is the Gorter-Mellink heat conductance. Strictly speaking, $S$ and $K$ are functions of temperature, but if they are taken constant, the pulsed-source problem has an analytic solution in plane geometry $(n=1){ }^{1}$ For this solution, the central temperature falls with elapsed time $t$ as $t^{-3 / 2}$.

The purpose of this paper is to explore the behavior of the central temperature in cylindrical $(n=2)$ and spherical $(n=3)$ geometries. To simplify the appearance of the equations, we work in special units in which $K=S=1$. 
Pulsed-source problem in spherical geometry

In spherical geometry, Eq. (1) takes the form

$$
\frac{\partial T}{\partial t}=\frac{1}{r^{2}} \frac{\partial}{\partial r}\left[r^{2}\left(\frac{\partial T}{\partial r}\right)^{1 / 3}\right]
$$

The boundary and initial conditions corresponding to the pulsed-source problem are

$$
\begin{aligned}
T(r, 0) & =0 \quad(r>0) \\
T(\infty, t) & =0 \\
\int_{0}^{\infty} 4 \pi r^{2} T d r & =Q \quad(t>0)
\end{aligned}
$$

The partial differential equation (2) is invariant to the family of affine groups

$$
\begin{aligned}
T^{\prime} & =\lambda^{\alpha} T \\
t^{\prime} & =\lambda^{\beta_{t}} \\
r^{\prime} & =\lambda r
\end{aligned}
$$

subject to the subsidiary relation

$$
2 \alpha-3 \beta=-4
$$

Tise boundary condition (3c) requires that $\alpha=-3$, so that $\beta=-2 / 3$. With these values, the similarity solution of Eq. (2) becomes ${ }^{2}$

$$
T=t^{9 / 2} y\left(r t^{3 / 2}\right)
$$

This last form is unsatisfactory because it represents a temperature distribution that peaks up as time goes on rather than one that spreads out. This defect is fundamental and means that the pulsed-source problem in spherical geometry has 
no solution of the form (5). It does, however, have solutions of a very closely related form, as we shall see next.

In addition to its invariance to the groups (4), Eq. (2) is invariant to the group of translations in time: $T^{\prime}=T, t^{\prime}=t+\lambda, r^{\prime}=r$. Solutions invariant to this group must be functions of the time difference $t-t_{0}$, where $t_{0}$ is some fiducial time that is determined by the boundary and initial conditions. These considerations suggest that we look for similarity solutions of the form

$$
T=\left(t_{0}-t\right)^{9 / 2} y\left[r\left(t_{0}-t\right)^{3 / 2}\right]
$$

If we substitute this form into Eq. (2), we obtain for $y$ the following ordinary differential equation:

$$
\frac{1}{\xi^{2}} \frac{d}{d \xi}\left(\xi^{2} \dot{y}^{1 / 3}\right)+\frac{3}{2} \xi \dot{y}+\frac{9}{2} y=0, \quad \varsigma=r\left(t_{0}-t\right)^{3 / 2}
$$

After multiplication by $\xi^{2}$, this can be integrated once to give

$$
\xi^{2} \dot{y}^{1 / 3}+\frac{3}{2} \xi^{3} y=C
$$

where $C$ is a constant of integration. If $y$ is regular at the origin $\xi=0$, then $C=0$. in which case Eq. (8) can be integrated a second time to give

$$
y=\left(a+\frac{27}{16} \xi^{4}\right)^{-1 / 2}
$$

where $a$ is a second constant of integration.

The solution (9) is an exact solution of the superfluid diffusion equation. Its overall form $c=\left(t_{0}-t\right)^{9 / 2} y\left[r\left(t_{0}-t\right)^{3 / 2}\right]$ was chosen so that the total heat $Q=$ $4 \pi \int_{0}^{\infty} r^{2} c d r$ would be conserved. But with the form (9) for $y$, the $Q$-integral diverges. Thus the solution (9) cannot fulfill the initial condition of a finite source pulse, and so. strictly spet'ing, the solution to the pulsed-source problem is not a similarity solution of the form (6). 
Suppose we consider a pulsed source in which the finite heat deposition is such that the initial temperature distribution is given by the similarity solution (9) out to a radius $r=R$, but is zero for $r>R$. If $R$ is large enough, the initial effect of the region $r>R$ on the temperature distribution near $r=0$ shouid be small. So the central temperature should begin falling like $\left(t_{0}-t\right)^{9 / 2}$. Since the region $r>R$ is initially colder than in the similarity solution (9), it seems plausible for the central temperature to continue falling faster than $\left(t_{0}-t\right)^{9 / 2}$. Indeed, we might expect that any temperature distribution that is initially smaller than some similarity solution of the family (9) will always remain smaller. Thus the central temperature should be bounded from above by $a^{-1 / 2}\left(t_{0}-t\right)^{9 / 2}$, which means it should vanish at some finite time after the pulse! The proof of these last assertions, given next, involves some complicated but rather standard analysis involving maximum and minimum principles.

\section{Proof of the foregoing assertions}

Let us consider the infinitesimal difference $\delta c$ between two neighboring solutions of the superfluid diffusion equation $c_{t}=r^{-2}\left(r^{2} c_{r}^{1 / 3}\right)$. It obeys the linear partial differential equation $(\delta c)_{t}=r^{-2}\left[r^{2}\left(c_{r}^{-2 / 3} / 3\right)(\delta c)_{r}\right]_{r}$, which has the form

$$
\frac{1}{r^{2}} \frac{\partial}{\partial r}\left(r^{2} k \frac{\partial \phi}{\partial r}\right)=\frac{\partial \phi}{\partial t}, k>0
$$

Equation (10) describes heat conduction in spherical geometry with a thermal conductivity $k$. The boundary and initial conditions that interest us are

$$
\begin{aligned}
\phi(r, 0) & >0 \\
\phi(\infty, t) & =0 \\
\phi \text { regular at } r & =0
\end{aligned}
$$


What we should like to show is that $\phi(r, t)>0$ for all $t>0$. In that case, two solutions $c_{1}$ and $c_{2}$ of the superfluid diffusion equation that obey the boundary conditions (11b) and (11c) and the initial condition $c_{1}>c_{2}$ are always ordered so that $c_{1}>c_{2}$.

We begin first by considering a function $\psi$ that obeys the strict differential inequality

$$
\frac{1}{r^{2}} \frac{\partial}{\partial r}\left(r^{2} k \frac{\partial \psi}{\partial r}\right)-\frac{\partial \psi}{\partial t}<0
$$

and the boundary condition (11c) in the rectangle $\Sigma$ in the (r,t)-plane (see Fig. 1). The minimum value of $\psi$ cannot lie in the interior of $\Sigma$, for if it did, say at a point $P$, then $\psi_{t}(P)=0, \psi_{r}(P)=0$, and $\psi_{r r}(P) \geq 0$. These conditions contradict the strict inequality (12). Moreover, the minimum value of $\psi$ cannot lie at an interior point $Q$ of $S_{4}$, for if it $\operatorname{did}$, then $\psi_{r}(Q)=0$ and $\psi_{r r}(Q) \geq 0$. Then from Eq. (12) it follows that $\psi_{t}(Q)>0$, which means that there must be yet smaller values of $\psi$ in the neighborhood of $Q$ inside $\Sigma$, contrary to hypothesis.

Finally, the minimum value of $\psi$ cannot lie at an interior point of $S_{1}$, but in order to prove this, we shall have to consider in detail the consequences of boundary condition (11c). First of all, any solution $c$ of the superfluid diffusion equation (2) that is regular at the origin must behave near $r=0$ like $a+b r^{4}+\ldots$ for only then can the right-hand side of Eq. (2) have a finite, nonzero limit as $r \rightarrow 0$. In that case $c_{r}^{-2 / 3} \sim r^{-2}$ near $r=0$ and thus so does $k$ in Eq. (10). Furthermore, $\psi$, being the infinitesimal difference between two solutions of the superfluid diffusion equation, also has the form $a+b r^{4}+\ldots$ near $r=0$. If we substitute this form for the first term on the left-hand side of Eq. (12) we find that it equals $12 b \cdot \lim _{r \rightarrow 0}\left(r^{2} k\right)$ at $r=0$. If the minimum of $\psi$ occurs at an interior point $T$ of $S_{1}$, then $\psi_{t}(T)=0$. Therefore $b(T)<0$, which means that there are yet smaller values of $\psi$ in the neighborhood of $T$ inside $\Sigma$, a contradiction. The minimum of $\psi$ must therefore lie on $S_{2}$ or $S_{3}$. 
When 0 satisfies the equality (10), we introduce the auxiliary function

$$
\psi=\phi+\epsilon t, \epsilon>0
$$

which satisfies the differential inequality (12). Furthermore, $\psi$, behaves like $a+b r^{4}+$ ... near $r=0$, as it should. Therefore, its minimum value in $\Sigma$ must lie on $S_{2}$ or $S_{3}$; call it $m_{\psi}$ and let it be attained at point $P$. Let $m_{\phi}$ be the smallest value $\phi$ attains on $S_{2}$ and $S_{3}$ (not yet proved to be its minimum value in $\Sigma$ !). Then from Eq. (13), for any point $Q$ in $\Sigma$,

$$
\phi(Q)+\epsilon t_{Q}=\psi(Q)>m_{\psi}
$$

and

$$
m_{\psi}=\psi(P)=\phi(P)+\epsilon t_{P} \geq m_{\phi}+\epsilon t_{P} .
$$

Thus, combining Eqs. (14) and (15), we find

$$
\phi(Q)>m_{\phi}+\epsilon\left(t_{P}-t_{Q}\right)
$$

Finally, if we let $\epsilon \rightarrow 0$, we see that $\phi$ is always $\geq$ the smallest value it attains on $S_{2}$ and $S_{3}$, so that its minimum must lie on $S_{2}$ or $S_{3}$.

If $\phi \geq 0$ on $S_{2}$ and $\phi=0$ on $S_{3}$, then its minimum value must be zero. Therefore, $\phi \geq 0$ everywhere in $\Sigma$, as was to be proved. The last step is to let $R \rightarrow \infty$.

\section{Discussion}

Suppose we establish an initial temperature rise $T_{1}$ inside a sphere of radius $R$. We can use the foregoing results to obtain an upper limit to the time at which the temperature rise disappears. To do so, we need to find a similarity solution whose initial form at $t=0$, namely, $T=t_{0}^{9 / 2}\left(a+27 r^{4} t_{0}^{6} / 16\right)^{-1 / 2}$, exceeds $T_{1}$ for $r \leq R$. The best (smallest) upper limit $t_{0}$ will correspond to an initial form $T$ that just 
equals $T_{1}$ at $r=R$ (see Fig. 2). The choices of $a$ and $t_{0}$ are therefore constrained by the relation

$$
T_{1}=t_{0}^{9 / 2}\left(a+27 R^{4} t_{0}^{6} / 16\right)^{-1 / 2}
$$

or

$$
t_{0}^{9}-\frac{27 R^{4} T_{1}^{2}}{16} t_{0}^{6}-a T_{1}^{2}=0 .
$$

Equation (18) has one real root, which is always larger than

$$
t_{0 *}=\frac{3}{2}\left(\frac{R^{4} T_{1}^{2}}{2}\right)^{1 / 3}
$$

and approaches $t_{0 *}$ as $a$ becomes smaller (see Fig. 3).

The radius $R$, the temperature rise $T_{1}$, and the source strength $Q$ are related by

$$
Q=\frac{4 \pi}{3} T_{1} R^{3}
$$

and if we use Eq. (20) to eliminate $R$ in Eq. (19), we obtain

$$
t_{0 *}=\frac{3}{2^{4 / 3}}\left(\frac{3}{4 \pi}\right)^{4 / 9} \cdot Q^{4 / 9} T_{1}^{2 / 9}
$$

The numerical constant in (21) equals $0.629889 \ldots$.

Equation (21) is correct in special units in which $K$ and $S$ are numerically equal to 1 . In ordinary units, Eq. (21) must be written

$$
t_{0 *}=0.629889\left(Q^{4} T_{1}^{2} S^{5}\right)^{1 / 9} / K
$$

When the bath temperature is $1.8 \mathrm{~K}$ (and the pressure is 1 atm), $S=$ $0.410 \mathrm{~J} \cdot \mathrm{cm}^{-3} \cdot \mathrm{K}^{-1}$ and $K=10.4 \mathrm{~W} \cdot \mathrm{cm}^{-5 / 3} \cdot \mathrm{K}^{-1 / 3}$. In the one-dimensional (1-D) experiment of Lottin and van Sciver, ${ }^{2}$ a heat pulse of $0.92 \mathrm{~J}$ was deposited at a point in a $6-\mathrm{mm}$ tube. Taling $T_{1}=T_{\lambda}-T_{b}=0.37 \mathrm{~K}$, we find, from Eq. (22), $t_{0 *}=28.5 \mathrm{~ms}$. So we expect the temperature rise to be extinguished in less than $30 \mathrm{~ms}$; this is quite different from the 1-D experiment of Lottin and van Sciver 
in which a $0.050 \mathrm{~K}$ temperature rise was still perceptible after one full second. This rather remarkable prediction has not yet been tested experimentally and is a tempting target for further study.

\section{Pulsed-source problem in cylindrical geometry}

In cylindrical geometry, Eq. (1) takes the form

$$
\frac{\partial T}{\partial t}=\frac{1}{r} \frac{\partial}{\partial r}\left[r\left(\frac{\partial T}{\partial r}\right)^{1 / 3}\right]
$$

We wish to solve it subject to the boundary and initial conditions

$$
\begin{aligned}
T(r, 0) & =0 \quad(r>0), \\
T(\infty, t) & =0 \\
\int_{0}^{\infty} 2 \pi r T d r & =Q \quad(t>0)
\end{aligned}
$$

Equation (23) is invariant to the mixed stretching-translation group

$$
\begin{aligned}
T^{\prime} & =\lambda^{-2} T \\
t^{\prime} & =t+\frac{1}{\beta} \ln \lambda \quad(0<\lambda<\infty) \\
r^{\prime} & =\lambda r
\end{aligned}
$$

where $\beta$ is an arbitrary constant not yet determined. The most general form for $T(r, t)$ invariant to a group of the family $(25)$ is

$$
T=\mathrm{e}^{-2 \beta t} y\left(r \mathrm{e}^{-\beta t}\right)
$$

where $y$ is an arbitrary function of the single argument $\xi=r e^{-3 t}$. Furthermore. Eq. (26) has the right form to satisfy the boundary condition (24c):

$$
2 \pi \int_{0}^{\infty} y(\xi) \xi d \xi=Q
$$


If we substitute Eq. (20) into Eq. (23), we get

$$
\frac{1}{\xi} \frac{d}{d \xi}\left(\xi \dot{y}^{1 / 3}\right)+\beta(2 y+\xi \dot{y})=0, \quad \xi=r \mathrm{e}^{-\beta t}
$$

After multiplication by $\xi$, this can be integrated to give

$$
\xi \dot{y}^{1 / 3}+\beta \xi^{2} y=C
$$

where $C$ is a constant of integration. If $y$ is regular at the origin $\xi=0$, then $C=0$. Then E.q. (29) can be integrated again to give

$$
y=\left(a+\frac{\beta^{3}}{2} \xi^{4}\right)^{-1 / 2}
$$

where $a$ is ancther constant of integration.

Again the $Q$-integral diverges, so the solution (30) cannot fulfill the initial condition of a finite heat pulse. But again, by a repetition of the argument of the last section, we expect it to exceed for all time any solution that it exceeds at $t=0$. Thus the central temperature should be bounded from above by $a^{-1 / 2} e^{-2 \beta t}$, which means it should fall exponentially with time.

As before, the choices of $a$ and $\beta$ in Eq. (30) are constrained by the initial condition

$$
T_{1}=\left(a+\frac{\beta^{3}}{2} R^{4}\right)^{-1 / 2}
$$

from which it follows that the largest possible value of $\beta$ is

$$
\beta_{*}=\left(\frac{2}{R^{4} T_{1}^{2}}\right)^{1 / 3}
$$

If we now define $t_{0 *}=1 / 2 \beta_{*}$, the relaxation time of the central temperature, we find

$$
t_{0 *}=\left(\frac{R^{4} T_{1}^{2}}{16}\right)^{1 / 3}
$$

The source strength $Q$ is related to $R$ and $T_{1}$ by

$$
Q=\pi R^{2} T_{1}
$$


so that

$$
t_{0 *}=\left(\frac{Q^{2}}{16 \pi^{2}}\right)^{1 / 3}
$$

Whereas in the spherical case $Q$ has the dimensions of $\mathrm{J}$, in the cylindrical case it has the dimensions of $\mathrm{J} \cdot \mathrm{m}^{-1}$. So in ordinary units,

$$
t_{0 *}=\frac{1}{\pi}\left(\frac{Q^{2} S}{16 \pi^{2}}\right)^{1 / 3}
$$

An energy deposition $Q$ of $1 \mathrm{~J} \cdot \mathrm{cm}^{-1}$ results in a relaxation time of $13.2 \mathrm{~ms}$.

\section{References}

1. Dresner, L., Advances in Cryogenic Engineering 29 (1984) 323.

2. Lottin, J.C., Van Sciver, S.W. Proc 9th Intrn Cryo Engr Conf, Butterworths (1982) 269.

\section{DISCLAIMER}

This report was prepared as an account of work sponsored by an agency of the United States Government. Neither the United States Government nor any agency thereof, nor any of their employees, makes any warranty, express or implied, or assumes any legal liability or responsibility for the accuracy, completeness, or usefulness of any information, apparatus, product, or process disclosed, or represents that its use would not infringe privately owned rights. Reference herein to any specific commercial product, process, or service by trade name, trademark, manufacturer, or otherwisc does not nccessarily constitutc or imply its endorsement, recommendation, or favoring by the United States Government of any agency thereof. The views and opinions of authors expressed herein do not necessarily state or reflect those of the United States Government or any agency thereof. 


\section{Figure captions}

Fig. 1 The rectangle in the $(r, t)$ plane used in proving the minimum principle for the differential inequality (12).

Fig. 2 A uniform initial temperature rise in the sphere of radius $R$ compared with the initial temperature distribution of a similarity solution.

Fig. 3 An auxiliary sketch depicting the relation of the real root of Eq. (18) to the coefficient $p$. 
ORNL.DWG 89M-2627 FED

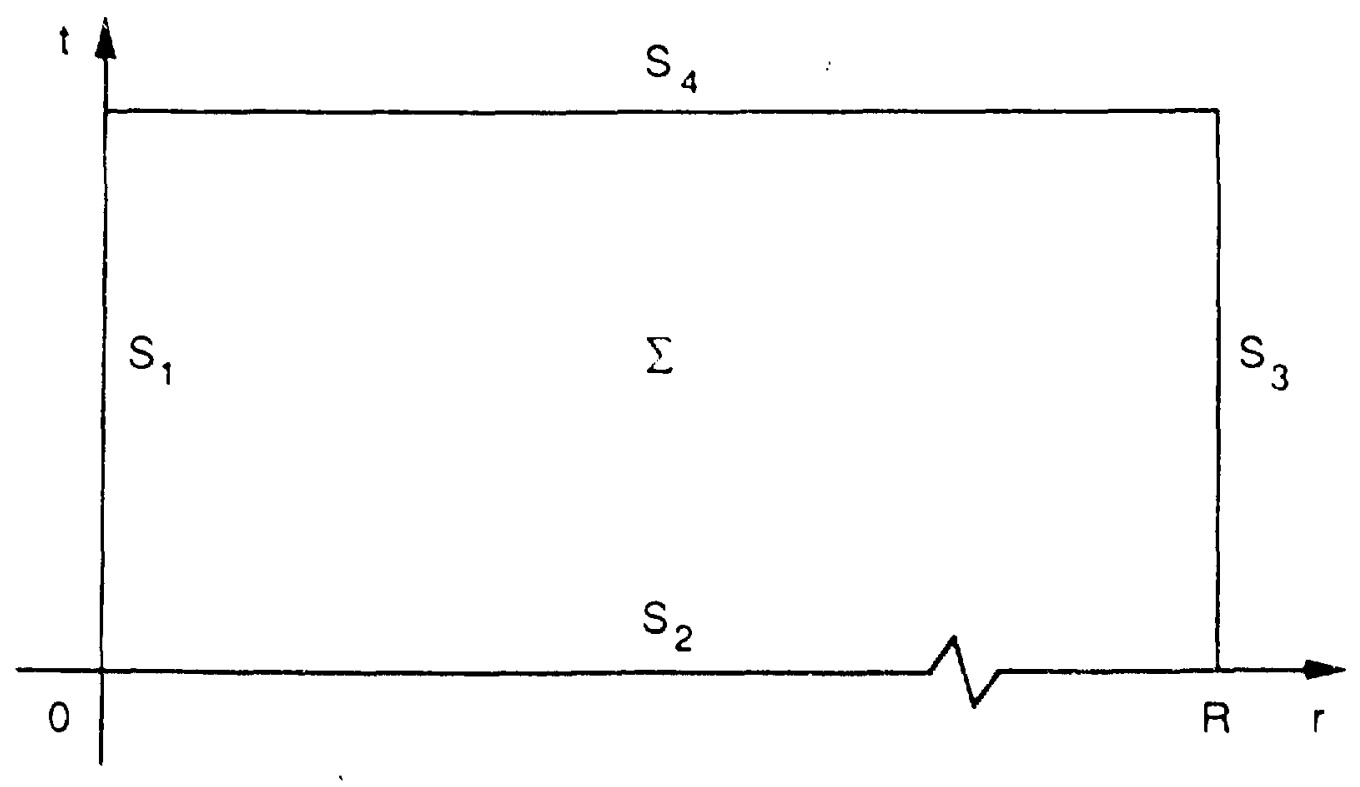


ORNI IWG $89 M 2628$ FED

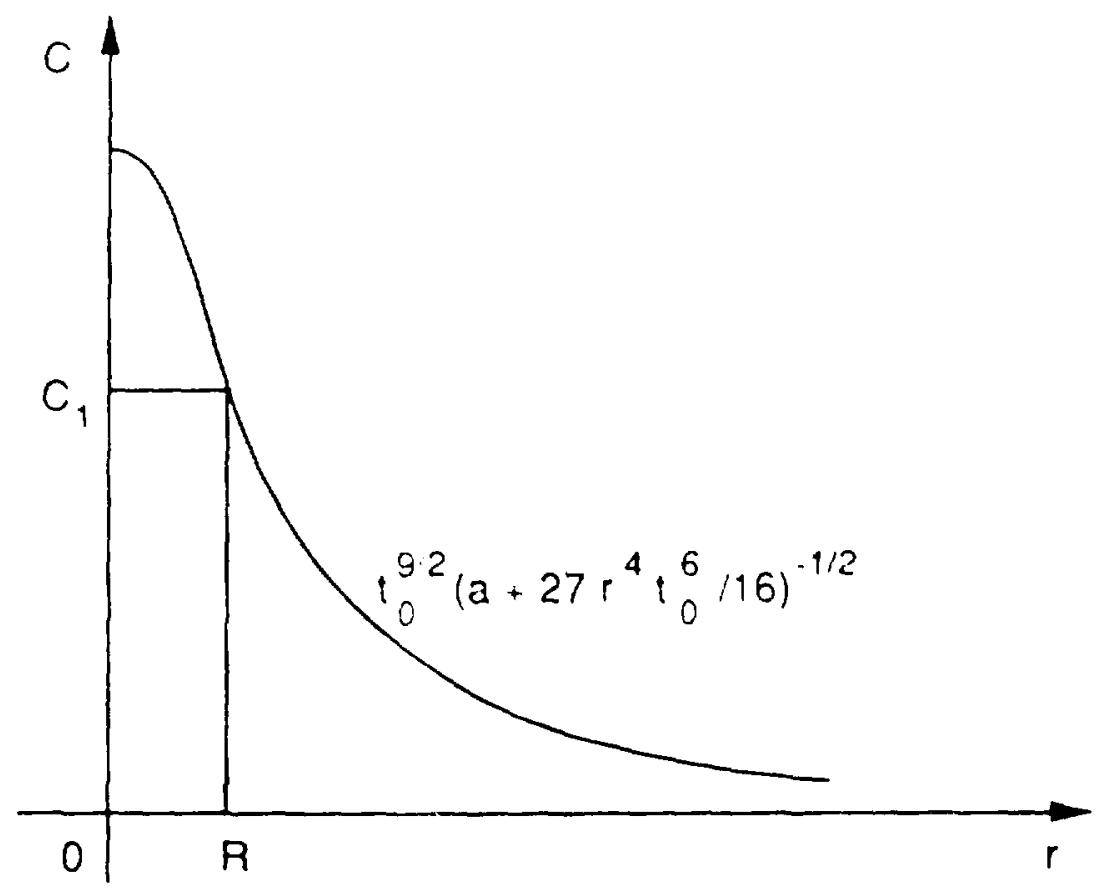


ORNL.OWG 89M-2629 FED

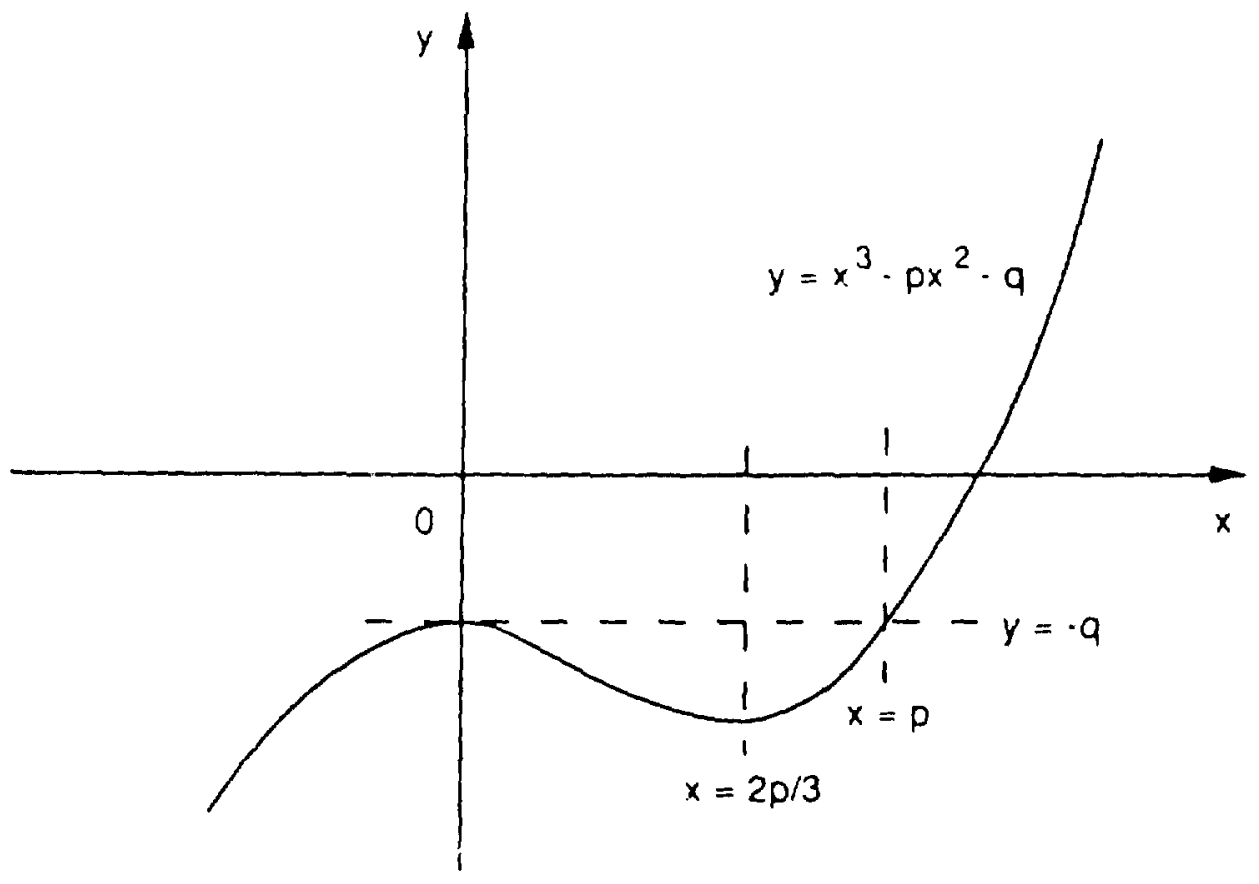

